\title{
バナッハ空間における最適追従問題†
}

\author{
舟橋康行 -中村嘉 平 \\ 名古屋大学工学部 名古屋市千種区不老町 \\ (昭和 43 年 9 月 10 日 受付)
}

\section{Optimal Pursuit Problem in Banach Spaces}

\author{
Yasuyuki Funahashi and Kahei Nakamura \\ (Faculty of Engineering, Nagoya University, Nagoya) \\ (Received September, 10, 1968)
}

\begin{abstract}
With the aid of functional analysis, the optimal pursuit problem and the optimal final value control problem in Banach spaces are considered. Many papers treat these problems in the Hilbert space setting and derive an adjoint equation and reduce to the solution of the two point boundary value problem. The authors pose the problems in the Banach spaces such as $L^{p}, 1 \leqq p \leqq+\infty$. In $L^{1}$ and $L^{\infty}$ spaces the norm is not Fréchet differentiable, not even Gateaux differentiable and admits one-sided Gateaux derivative only.

A necessary and sufficient condition for an optimal control is derived using one-sided Gateaux derivative. The equations of the optimal condition in $L^{1}, L^{p}(1<p<+\infty)$ and $L^{\infty}$ spaces are obtained in the explicit form. And finally several examples are illustrated in the Hilbert space setting in order to make this theory understand with ease. The method is straightforward and thus the optimal control can be computed directly.
\end{abstract}

\section{1. 緒言}

本稿の目的は，関数解析の手法により分布定数系の 最適制御問題を解くことである。筆者らはさきに，半 群の理論を用いれば分布定数系は集中定数系と同一の 形でダイナミカル表示されることを示し，その利点が 集中定数系について展開された制御理論が自然の形で 分布定数系にまでも抎張できることであることを指摘 した ${ }^{1), 2)}$. 本稿ではバナッ八空間に执いて最適制御問 題を考察することによりこの点を明確にする。

分布定数系の最適制御問題に対する解法は, 最大原 理によるもの ${ }^{3), 4)}$, ダイナミックプログラミングによ

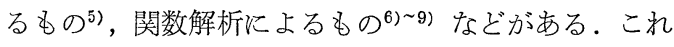
らのらち Fattorini ${ }^{6)}$ は最短時間制御問題を, Balakrishnan ${ }^{7)}$, Friedman $^{8)}$ は最短時間和よび最終值制御 問題を，Axelband9は追従問題をそれぞれバナッハ 空間で考察している。

本稿では追従問題䍩よび最終值制御の変形した問題
を考察する。これらの問題はすで多くの論文で扱わ れているが，そのほとんどが共役 (adjoint) 方程式を 誘導して 2 点境界值問題を解くことに帰着させてい $3^{4), 9,, 10)}$. 本稿は『凸関数の最小值はその導関数がゼ 口になる点で得られる』という概念を関数空間に拡張 して最適解の必要十分条件を導く。

追従問題はヒルベルト空間で設定されて解かれてき た、筆者らる “ヒルベルト空間のノルムはフレッシェ 微分可能 (Fréchet differentiable) である”というこ とによって，ヒルベルト空間での追従問題を解いた。 本稿ではさらに一般の $L^{p}(1 \leqq p \leqq+\infty)$ なるバナッ八 空間での追従問題をも考察する. そのために, 片側が トウ微分 (one-sided Gateaux differential) を導入す る.なぜならば, $L^{p}(1<p<+\infty)$ なるバナッ八空間 のノルムは原点以外ではフレッシェ微分可能である ${ }^{12)}$ が, $L^{1}$ や $L^{\infty}$ のノルムはフレッシェ微分可能でもな くまたガトウ微分可能でもないからである，最後比 ルベルト空間での例をあげその最適解を計算する。

$†$ 第 6 回計測自動制御学会学術講演会で一部発表 (昭 42 .

10) 


\section{2. システムの記述と問題の定式化}

問題を一般的に定式化するまえに，あとで考察する 例をあげて具体的な問題括よび記号の説明をする。

まずつぎの式で記述される熱系を考える。

$$
\begin{gathered}
\frac{\partial}{\partial t} u(t, x)=\frac{\partial^{2}}{\partial x^{2}} u(t, x)+f(t, x) \text { a.e.in } t, \\
0<x<1
\end{gathered}
$$

$$
\text { 境界条件 } \quad u(t, 0)=u(t, 1)=0
$$

ここに熱伝導係数が 1 になるように正規化してある。 システムの初期状態が $u(0, x)$ であるとき， $(0, T)$ 区間で与えられた希望状態の軌道 $u^{\vec{a}}(t, x)$ にシステム を追従させる入力 $f$ ，すなわちつぎの評価関数を最小 にする制御入力 $f$ を決定する問題——最適追従問題と 呼ぶ——考える。

$$
\begin{aligned}
J(f) & =\frac{1}{2} \int_{0}^{T} \int_{0}^{1}\left|u(t, x)-u^{d}(t, x)\right|^{2} d x d t \\
& +\frac{1}{2} \int_{0}^{T} \int_{0}^{1}|f(t, x)|^{2} d x d t
\end{aligned}
$$

（2）式から（1）式の解 $u(t, x)$ は各時刻 $t$ で $L^{2}$ (S) に属していることが要請されている.ただし $L^{2}$

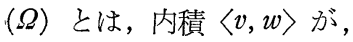

$$
\langle v, w\rangle=\int_{\Omega} v(x) w(x) d x, \Omega=(0,1)
$$

で定義された 2 乗可積分な実数值関数からなるヒルベ ルト空間である。

$$
\text { ここで, } u(t) \equiv u(t, \cdot), f(t) \equiv f(t, \cdot) \text { といら } L^{2}(\Omega)
$$

を值にとる関数を定義し，

$$
\begin{aligned}
& A=\frac{\partial^{2}}{\partial x^{2}} \text {, 定義域 } \mathfrak{D}(A) \\
& =\left\{\begin{array}{l|l}
v \in L^{2}(\Omega) & \begin{array}{c}
v, v^{\prime} \text { が絶対連続で } \\
v^{\prime \prime} \in L^{2}(\Omega) \\
v(0)=v(1)=0
\end{array}
\end{array}\right\}
\end{aligned}
$$

とすれば (1)，（2）式はつぎのように書かれる.

$$
\begin{gathered}
\frac{d}{d t} u(t)=A u(t)+f(t) \\
J(f)=\frac{1}{2} \int_{0}^{T}\left\|u(t)-u^{d}(t)\right\|^{2}{ }_{L^{2}(\Omega)} d t \\
+\frac{1}{2} \int_{0}^{T}\|f(t)\|^{2}{ }_{L^{2}(\Omega)} d t
\end{gathered}
$$

さらに, $(0, T)$ 上で定義され $L^{2}(\Omega)$ を值にとる 2 乗可積分な関数 $g$, すなわち,

$$
\begin{aligned}
& g(t) \in L^{2}(\Omega) \text { a. e. in } t \in(0, T) \text { でかつ, } \\
& \int_{0}^{T}\|g(t)\|^{2} L^{2}(\Omega) d t<+\infty
\end{aligned}
$$

なる関数の集合に，

$$
\langle g, h\rangle=\int_{0}^{T}\langle g(t), h(t)\rangle_{L^{2}(\Omega)} d t
$$

$$
\|g\|\left\|^{2}=\int_{0}^{T}\right\| g(t) \|^{2} L^{2}(\Omega) d t
$$

なる内積とンルムを導入した線形空間を $L^{2}\left(0, T ; L^{2}\right.$ $(\Omega) ）$ と記す. $L^{2}\left(0, T ; L^{2}(\Omega)\right)$ はヒルベルト空間で あり，この記号を用いれば (3) 式はつぎの形に書く ことができる。

$$
\begin{aligned}
& \frac{d}{d t} u(t)=A u(t)+f(t) \\
& J(f)=\left.\frac{1}{2}\left\|u-u^{d}\right\|\right|^{2}+\frac{1}{2}\||f|\|^{2}
\end{aligned}
$$

よって, (1),(2) 式で記述される最適追従問題は けっきょく（3），(5) 式の形で述べることができた。

同じ熱系についてもら1つの制御問題を考学る。初 期状態が $u(0, x)$ なるとき, 最終時刻 $T$ での状態を希 望状態 $u^{d}(T, x)$ に近づる入力 $f$, すなわちつぎの 評価関数を最小にする制御入力 $f$ を決定する問題— 最終値制御問題と呼ぶー一である。

$$
\begin{aligned}
I(f) & =\frac{1}{2} \int_{0}^{1}\left|u(T, x)-u^{d}(T, x)\right|^{2} d x \\
& +\frac{1}{2} \int_{0}^{T} \int_{0}^{1}|f(t, x)|^{2} d x d t
\end{aligned}
$$

このときにも $u(t, \cdot) \in L^{2}(\Omega)$ が要請され, かつ $f \in$ $L^{2}\left(0, T ; L^{2}(\Omega)\right)$ でなければならない. $L^{2}(\Omega), L^{2}(0$, $\left.T ; L^{2}(\Omega)\right)$ のノルムを用いて書きなおせぜ，

$$
\begin{aligned}
& \frac{d}{d t} u(t)=A u(t)+f(t) \\
& I(f)=\frac{1}{2}\left\|u(T)-u^{d}(T)\right\|^{2} L^{2}(\Omega)+\frac{1}{2}\|f\|^{2}
\end{aligned}
$$

となる. 以上 2 つ例から最適追従問題と最終值制御 問題はヒルベルト空間のとり方が異なるのみで理論的 にはまったく同一の枠内で扱いらることがわかった。

以上の例を一般化してつぎのように問題を定式化す る。

バナッハ空間で記述されたダイナミカルシステム

$$
\frac{d}{d t} u(t)=A u(t)+B f(t)
$$

初期状態は $u(0)$ に対して, 評価関数（9）式また は (10) 式を最小にする制御入力 $f \in L^{p}(0, T ; F)$ を 決める。

ここに, $u(t)$ は時刻 $t$ での状態で, 各 $u(t)$ はバナ ッ八空間 $U$ に属する。 $f(t)$ は時刻 $t$ での制御で，各 $f(t)$ はバナッハ空間 $F$ に属する。 $A$ は定義域 $\mathfrak{D}(A)$ がUで稠密な線形閉作用素， $B$ は $F$ から $(A) \subset U$ へ の線形作用素である.

$$
J(f)=\left\|u-u^{d}\right\|+\|f\|
$$

* （8）式の等号の意味は，一般には $U$ より弱い位相に関 するものである.詳細は文献（2）を参照されたい. 


$$
I(f)=\left\|u(T)-u^{d}(T)\right\|+\|f\|
$$

ここに, $\|u\|,\|u(T)\|,\|f\|$ の各ノルムはそれぞれ $L^{q}(0, T ; U) q>1, U$ 扤よび $L^{p}(0, T ; F) p \geqslant 1$ のノル ムをあらわす。

Balakrishnan6) 飞よれば, $A$ が強連続な半群 $\{S(t)$ ， $t \geqslant 0\}$ の生成作用素であるときとは，

$B f(t) \in \mathscr{D}(A)$ a.e. in $t$ から $A B f \in L^{1}(0, T ; U)$ なる入力 $f$ に対して初期状態 $u(0) \in \mathscr{D}(A)$ の下で

（8）式の解は一意に存在して次式で書かれる.

$$
u(t)=S(t) u(0)+\int_{0}^{t}(S(t-\sigma) B f(\sigma) d \sigma
$$

また，とくに $A$ が放物形半群の生成作用素であると きには，解が一意に存在して (11) 式で書くことので きる条件をつぎのように述べることができる11.

$$
f \in L^{1}(0, T ; F) \text { かつ } u(0) \in U
$$

さて以上のことをもとにして（9)，(10) 式を書き な拈し，2つの最適制御問題が同一の枠内で取扱いら ることを示す。

$$
\left(K_{1} f\right)(t)=\int_{0}^{t} S(t-\sigma) B f(\sigma) d \sigma, \quad 0<t<T
$$

で $L^{p}(0, T ; F)$ から $L^{q}(0, T ; U)$ への有界作用素を 定義し，

$$
c_{1}(t)=u^{d}(t)-S(t) u(0), \quad 0<t<T
$$

とすれば（9）式は，

となる。をた、

$$
J(f)=\left\|K_{1} f-c_{1}\right\|+\|f\|
$$

$$
K_{2} f=\int_{0}^{T} S(T-\sigma) B f(\sigma) d \sigma
$$

で $L^{p}(0, T ; F)$ からUへの有界作用素を定義し，

$$
c_{2}=u_{d}(T)-S(T) u(0)
$$

とすれば，(10) 式は（9）式と同様に，

$$
I(f)=\left\|K_{2} f-c_{2}\right\|+\|f\|
$$

と書くことができる。すなわち（9），(10）式で提起 した最適追従問題と最終值制御問題は (12) 式の形の 評価関数を最小にすること㯰き換えられた。よって 以後は (12) 式の評価関数を最小にすることを考光る。

\section{3. 最適解の必要十分条件}

許容制御入力 $f$ として $L^{1}$ または $L^{\infty}$ の空間を考光 るときには, $J(f)$ はその空間でフレッシェ微分可能 ではない，ここでは片側ガトウ微分の考学から最適解 の必要十分条件を導く。

$J(f)$ はノルムの性質により凸関数である。じっさ い, $0<\alpha<1$ なる実数 $\alpha$ と, 任意の $g, f \in L^{p}(0, T ; F)$ に対して,

$$
\begin{aligned}
& J(\alpha f+(1-\alpha) g)=\|K(\alpha f+(1-\alpha) g)-c\| \\
& \quad+\|\alpha f+(1-\alpha) g\|
\end{aligned}
$$

$$
\begin{aligned}
& =\|\alpha(K f-c)+(1-\alpha)(K g-c)\| \\
& +\|\alpha f+(1-\alpha) g\| \\
& \leqq \alpha J(f)+(1-\alpha) J(g)
\end{aligned}
$$

となる。

さて，fが最適解であるための必要十分条件はつぎ の式で与えられる.

$$
J(f+\epsilon g)-J(f) \geqslant 0 \quad \forall \varepsilon>0 \quad \forall g \in L^{p}(0, T ; F)
$$

ここで,

$$
V(\epsilon)=(J(f+\epsilon g)-J(f)) / \varepsilon, \quad \varepsilon>0
$$

と怙けば $V(\epsilon) \geqslant 0$ である. 在すればとうぜん, $\lim _{\epsilon \downarrow 0} V(\epsilon) \geqq 0$ である. $J(f)$ が凸 関数であることから $V(\epsilon)$ は $\varepsilon \downarrow 0$ について単調減少 となる。したがって “有界な単調減少列は収束する” という解析学でよく知られた定理により, $\lim _{\epsilon \downarrow 0} V(\epsilon) の$ 存在が保証される13)。このことをつぎの定理にまとめ る.

【定理 1】 $f$ が最適解であるための必要十分条件は， (15) 式がすべての $g \in L^{p}(0, T ; F)$ について成立す ることである.

$$
\delta^{+} J(f: g)=\lim _{\epsilon \downarrow 0} \frac{J(f+\epsilon g)-J(f)}{\epsilon} \geqslant 0
$$

【証明】十分条件 $\quad V(\epsilon)$ は $\varepsilon \downarrow 0$ について単調 減少列であるから，

$$
V(1) \geqq V(0+)=\delta^{+} J(f: g)
$$

である。

$$
\begin{gathered}
J(f+g)-J(f) \geqq \delta^{+} J(f: g) \geqslant 0 \forall g \\
\therefore \quad J(f+g) \geqq J(f) \quad \forall g \in L^{p}(0, T ; F)
\end{gathered}
$$

すなわち $f$ は $J$ 最小元である。

必要条件 ある $g_{0} \neq 0$ 汶対して $\delta^{+} f\left(f: g_{0}\right)<0$ で あると仮定する。 $V(\epsilon)$ は $\varepsilon>0$ について連続である （付録 I）から，十分小さい $\varepsilon_{0}$ に対して，

$$
V(\epsilon) \leqq V\left(\epsilon_{0}\right)<0 \quad \forall 0<\varepsilon \leqq \varepsilon_{0}
$$

が成立する。

$$
\therefore J\left(f+\epsilon g_{0}\right)<J(f) \quad \forall 0<\epsilon \leq \varepsilon_{0}
$$

となり, $f$ の最適性に反する。（証明終）

$\delta^{+} J(f: g)$ は $f$ 飞和故る $g$ 方向の片側ガトウ微分 (one-sided Gateaux differential) と呼ばれる. $\delta^{+} J$ $(f: g)$ はつぎの性質をもっている.

(i) 正値同次性 (positive homogenvity) $\delta^{+} J(f: \lambda g)=\lambda \delta^{+} J(f: g), \quad \forall \lambda \geqq 0$

(ii) 劣加法性 (subadditivity) $\delta^{+} J(f: g+h)$ $\leqq \delta^{+} J(f: g)+\delta^{+} J(f: h)$

さらに $\delta^{+} J(f: g)$ がつぎの式,

$$
\delta^{+} J(f:-g)=-\delta^{+} J(f: g)
$$

を満足するとき， $\delta^{+} J(f: g)$ は $g$ について同次となる。 
このとき，Jは $f$ でガトウ微分可能であるといい, こ れを $\delta J(f: g)$ と記す ${ }^{13)}$.

【系】 Jがガトウ微分可能であるとき, Jが $f$ で最 小となるための必要十分条件は，

$$
\delta J(f: g)=0 \quad \forall g \in L^{p}(0, T ; F)
$$

である。

つぎに最適制御の一意性をしらべる。

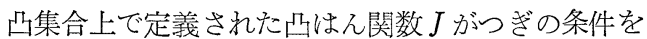
又たすとき，狭義凸はん関数 (strictly convex functional) と呼ばれる14).

$g \neq f$ なるとき，

$J(\alpha f+(1-\alpha) g)<\alpha J(f)+(1-\alpha) J(g) \quad \forall 0<\alpha<1$ この狭義凸はん関数に対してはつぎの定理が成立する。

【定理 2】 $J(f)$ が狭義凸は九関数であるとき，最 適解が存在すればそれは一意である。

【証明】 $f_{1}, f_{2}\left(f_{1} \neq f_{2}\right)$ が $\min _{f} J(f)=\beta$ を与光る 2 つの最適解とする。このとき， $0<\alpha<1$ なる $\alpha$ 亿対し $\tau$,

$J\left(\alpha f_{1}+(1-\alpha) f_{2}\right)<\alpha J\left(f_{1}\right)+(1-\alpha) J\left(f_{2}\right)=\beta$ となる。 $f_{1}, f_{2}$ の最適性に反する。よって最適解は一 意である。（証明終)

\section{4. 各種の空間での必要十分条件}

バナッ八空間 $F$ 和よび $p, q$ を定めて (15) 式を具体 的什計算する.4.1, 4.2,4.3 では希望状態とシステム の状態との差はとルベルトノルムではかるるのとする.

\section{$4 \cdot 1$ 消費燃料に注目する場合}

$F$ は実数（スカラー）空間とする。制御入力 $f$ を,

$$
\|f\|_{1}=\int_{0}^{T}|f(t)| d t
$$

なる $L^{1}$ ノルムで評価する。このとき評価関数，

$$
J_{1}(f)=\|f\|_{1}+\frac{1}{2}\|K f-c\|_{2}{ }^{2}
$$

を最小にする制御入力 $f$ を求める.

$$
\begin{aligned}
& Z(f)=\{t \mid 0 \leq t \leq T, \quad f(t)=0\} \\
& \operatorname{sgn} f(t)=\left\{\begin{array}{cc}
f(t) /|f(t)| & f(t) \neq 0 \\
0 & f(t)=0
\end{array}\right.
\end{aligned}
$$

と定義すれば, $\delta^{+} J_{1}(f ; g)$ は付録 II, IV から，

$$
\begin{aligned}
\delta^{+} J_{1}(f ; g) & =\lim _{\epsilon \downarrow 0} \frac{\|f+\epsilon g\|_{1}-\|f\|_{1}}{\epsilon} \\
& +\frac{1}{2} \frac{\|K f-c+\epsilon K g\|_{2}{ }^{2}-\|K f-c\|_{2}{ }^{2}}{\epsilon} \\
& =\int_{Z(f)}|g(t)| d t+\langle\operatorname{sgn} f
\end{aligned}
$$

*1 ここではヒルベルトノルムは2 乗してある. それでない と得られる条件式が複雑になり実際の計算が非常に困難 になる. 以下рノルムの場合には 乗して評価する。な

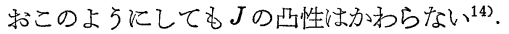

$$
\left.+K^{*} K f-K^{*} c, g\right\rangle \geqq 0^{* 2}
$$

同様に，

$$
\begin{aligned}
\delta^{+} J(f:-g) & =\int_{Z(f)}|g(t)| d t-\langle\operatorname{sgn} f \\
& \left.+K^{*} K f-K^{*} c, g\right\rangle \geqq 0
\end{aligned}
$$

上の 2 つ式から最適解 $f$ のみたず゙き式が得られる.

$$
\left|\left\langle\operatorname{sgn} f+K^{*} K f-K^{*} c, g\right\rangle\right| \leqq \int_{Z(f)}|g(t)| d t
$$

$R(f)=\{t \in(0, T) \mid f(t) \neq 0\}$ と和くとき, $Z(f) \cap R(g)$ のルベーク測度が 0 となる $g$ に対して (19) 式は,

$$
\left\langle\operatorname{sgn} f+K^{*} K f-K^{*} c, g\right\rangle=0
$$

となる。したがって，とくに $f$ の゙ロ点が高々可算個 である場合には最適解 $f$ はつぎの式で与兄られる。

$$
\operatorname{sgn} f+K^{*} K f-K^{*} c=0
$$

$F$ が一般の $L^{1}$ または $n$ 次元ニークリッド空間 $R^{n}$ のとさには付録 II を参照して同様の条件式を得るこ とができる。

\section{$4 \cdot 2$ 消費エネルギに注目する場合}

$F$ はヒルベルト空間とし, 制御入力 $f$ は,

$$
\|f\|_{2}^{2}=\int_{0}^{T}\|f(t)\|_{F}^{2} d t
$$

で評価する。このとき評価関数,

$$
J_{2}(f)=\frac{1}{2}\|f\|_{2}{ }^{2}+\frac{1}{2}\|K f-c\|_{2}{ }^{2}
$$

を最小にする最適制御を求める。

$$
\begin{aligned}
\delta^{+} J_{2}(f ; g) & =\lim _{\epsilon \downarrow 0} \frac{1}{2}\left(\frac{\|f+\epsilon g\|_{2}{ }^{2}-\|f\|_{2}{ }^{2}}{\epsilon}\right. \\
& +\frac{\|K f-c+\epsilon K g\|_{2}{ }^{2}-\|K f-c\|_{2}{ }^{2}}{\epsilon} \\
& =\langle f, g\rangle_{L^{2}(0, T ; F)}+\langle K f-c, K g\rangle_{L^{2}(0, T: U)}{ }^{* 3} \\
& =\left\langle f+K^{*} K f-K^{*} c, g\right\rangle_{L^{2}(0, T ; F)}
\end{aligned}
$$

$\delta^{+} J_{2}(f ;-g)=-\delta^{+} J_{2}(f: g)$ となり, $J_{2}(f)$ はガトウ 微分可能である。したがって定理 1 の系より必要十分 条件はつぎの式で与兄られる。

$$
\begin{aligned}
f+K^{*} K f-K^{*} c & =0 \\
{\left[I+K^{*} K\right] \quad f } & =K^{*} c
\end{aligned}
$$

$\left[I+K^{*} K\right]$ の逆作用素吕存在してから有界作用素と なる（付録 III）から，最適解の存在も保証される。

$$
f=\left[I+K^{*} K\right]^{-1} K^{*} c
$$

さらに $\left[I+K K^{*}\right]^{-1}$ も存在して,

$$
\left[I+K^{*} K\right]^{-1} K^{*}=K^{*}\left[I+K K^{*}\right]^{-1}
$$

となるから（24）式はつぎの式に書くこともできる。

*2 記号く,〉はつぎのことをあらわす。

$$
\langle f, g\rangle=\int_{0}^{T} f(t) g(t) d t
$$

*3 $\langle f, g\rangle_{L} 2(0, T ; F)=\int_{0}^{T}\langle f(t), g(t)\rangle_{F} d t$ なる内積を意味す る. 


$$
f=K^{*}\left[I+K K^{*}\right]^{-1} C
$$

\section{$4 \cdot 3$ 制御入力の振幅に注目する場合}

$F$ は実数空間とし， $f$ は $(0, T)$ 上で定義された有 界関数とする，そして制御入力 $f$ を，

$$
\|f\|_{\infty}=\sup _{0 \leq t \leq T}|f(t)|
$$

なるノルムで評価するものとする。このとき評価関数，

$$
J_{\infty}(f)=\|f\|_{\infty}+\frac{1}{2}\|K f-c\|_{2}^{2}
$$

を最小とする最適入力 $f$ の満たすべき式を求める. $\delta^{+} J_{\infty}(f: g)$ は付録 $\mathbf{V}$ を参照すれば,

$$
\begin{aligned}
\delta^{+} J_{\infty}(f: g) & =\lim _{\epsilon \downarrow 0}\left\{\frac{\|f+\epsilon g\|_{\infty}-\|f\|_{\infty}}{\epsilon}\right. \\
& \left.+\frac{1}{2} \frac{\|K f-c+\epsilon K g\|_{2}^{2}-\|K f-c\|_{2}^{2}}{\epsilon}\right\} \\
& =\sup _{\epsilon>0}\left[\inf _{\|f\|_{\infty}-f(t) \mid<\epsilon} f(t) g(t)\right] \\
& +\left\langle K^{*} K f-K^{*} c, g\right\rangle
\end{aligned}
$$

同様に $\delta^{+} J_{\infty}(f ;-g)$ を計算し，両者が非負であるこ とから, 最適制御 $f$ はつぎの式で与えられる。

$\inf _{\epsilon>0}\left[\operatorname{Lup}_{\|f\|_{\infty}-f(t) \mid<\epsilon} f(t) g(t)\right] \leqq\left\langle K^{*} c-K^{*} K f, g\right\rangle$

$$
\leqq \sup _{\varepsilon>0}\left[\inf _{\left|\|f\|_{\infty}-f(t)\right|<\epsilon} f(t) g(t)\right]
$$

ここに $g$ は有界関数である.

とくに $g$ が連続関数であるときには，(28) 式は， $\min _{t \cdot|f(t)|=\|f\|_{\infty}} f(t) g(t) \leqq\left\langle K^{*} c-K^{*} K f, g\right\rangle$

$$
\leqq \max _{t:|f(t)|=\|f\|_{\infty}} f(t) g(t)
$$

となる。

4.4 一般のバナッハ空間の場合 $(1<\boldsymbol{p}, \boldsymbol{q}<+\infty)$

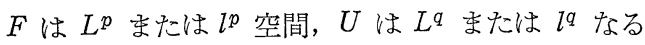
空間とする。評価関数,

$$
J_{p, q}(f)=\frac{1}{p}\|f\|_{p}{ }^{p}+\frac{1}{q}\|K f-c\|_{q^{q}}
$$

を最小にする $f$ の条件式を求める。

$$
\begin{aligned}
\delta^{+} J_{p, q}(f ; g) & =\lim _{\epsilon \downarrow 0} \frac{J_{p, q}(f+\epsilon g)-J_{p, q}(f)}{\epsilon} \\
& =\left\langle|f|^{p-1} \operatorname{sgn} f, g\right\rangle \\
& +\left\langle|K f-c|^{q-1} \operatorname{sgn}(K f-c), K g\right\rangle
\end{aligned}
$$

$J_{2}$ と同様に (30) 式はガトウ微分可能であるから, 最 適制钭は次式で与えられる。

$$
|f|^{p-1} \operatorname{sgn} f+K^{*}|K f-c|^{q-1} \operatorname{sgn}(K f-c)=0
$$

な执 $\|f\|^{p}$ が狭義凸関数であることから $J_{p, q}$ も狭 義凸関数となり, 定理 2 にり最適制御は一意である.

\section{5. 例}

ヒルベルト空間での最適追従問題および最終值制御
問題の例をあげ，作用素とその共役作用素 $K^{*}$ の具体 的な形，执よび最適解の計算方法を示す.

\section{$\mathbf{5} \cdot \mathbf{1}$ 空間分布制御入力による最終值制御}

$$
\left.\begin{array}{l}
\frac{\partial}{\partial t} u(t, x)=\frac{\partial^{2}}{\partial x^{2}} u(t, x)+f(t, x) \quad 0<x<1 \\
\text { 境界条件 } u(t, 0)=0, u(t, 1)=0
\end{array}\right\}
$$

初期状態が $u(0, x)$ であるときつぎの評価関数,

$$
\begin{aligned}
I(f) & =\frac{1}{2} \int_{0}^{1}\left|u(T, x)-u^{d}(T, x)\right|^{2} d x \\
& +\frac{1}{2} \int_{0}^{T} \int_{0}^{1}|f(t, x)|^{2} d x d t
\end{aligned}
$$

を最小にする最適制御 $f$ を決定する。

2. で示したように, $u(t) \equiv u(t, \cdot), f(t)=f(t, \cdot)$ その 他の記号を適切に約束すれば (32) 式は次式になる。

$$
\dot{u}(t)=A u(t)+f(t)
$$

$A$ から生成される半群 $S(t) t \geqslant 0$ を用いれば, $f \in L^{2}$ $\left(0, T ; L^{2}(\Omega)\right) \Omega=(0,1)$ なる入力に対して解は，

$$
u(t)=S(t) u(0)+\int_{0}^{t} S(t-\sigma) f(\sigma) d \sigma
$$

と書かれる。ここに，

$$
S(t) u=\sum_{n=1}^{\infty} e^{\lambda_{n} t} u_{n} \phi_{n}
$$

$\phi_{n}(x)=\sqrt{2} \sin n \pi x, \lambda_{n}=-(n \pi)^{2}, n=1,2, \cdots$

$$
u_{n}=\int_{0}^{1} u(x) \phi_{n}(x) d x
$$

このとき，

$$
K_{1} f=\int_{0}^{T} S(T-\sigma) f(\sigma) d \sigma
$$

で $L^{2}\left(0, T ; L^{2}(\Omega)\right)$ から $L^{2}(\Omega)$ への有界作用素 $K_{1}$ を 定義する. $K_{1}$ の共役作用素 $K_{1}$ * $L^{2}(\Omega)$ から $L^{2}$ $\left(0, T ; L^{2}(\Omega)\right)$ への有界作用素であり, 次式で定義さ れる。

$$
\left\langle K_{1} f, u\right\rangle_{L^{2}(\Omega)}=\left\langle f, K_{1}^{*} u\right\rangle_{L^{2}\left(0 \cdot T ; L^{2}(\Omega)\right)} \forall f
$$

ここに,

$$
\begin{aligned}
\left\langle K_{1} f, u\right\rangle & =\left\langle\int_{0}^{T} S(T-\sigma) f(\sigma) d \sigma, u\right\rangle \\
& =\int_{0}^{T}\langle S(T-\sigma) f(\sigma), u\rangle d \sigma \\
& =\int_{0}^{T}\left\langle f(\sigma), S^{*}(T-\sigma) u\right\rangle_{L^{2}(\Omega)} d \sigma
\end{aligned}
$$

いっぽら，

$$
\left\langle f, K_{1} * u\right\rangle=\int_{0}^{T}\left\langle f(\sigma),\left(K_{1}^{*} u\right)(\sigma)\right\rangle_{L^{2}(\Omega)} d \sigma
$$

であり，む $S^{*}(t)=S(t)$ であるから，

$$
\left(K_{1}^{*} u\right)(t)=S(T-t) u \quad 0<t<T
$$

となる。

$c=u^{d}(T)-S(T) u(0)$ と特忖ば最適解 $f^{\circ}$ は (25) 式加 ,

$$
f^{\circ}=K_{1} * v, \quad v=\left[I+K_{1} K_{1} *\right]^{-1} c
$$


で与えられる。

$$
v+K_{1} K_{1} * v=c
$$

まず $K_{1} K_{1} * v$ を計算する。

$$
\begin{aligned}
K_{1} K_{1} * v & =\int_{0}^{T} S(T-\sigma)\left(K_{1}^{*} v\right)(\sigma) d \sigma \\
& =\int_{0}^{T} S(T-\sigma) S(T-\sigma) v d \sigma \\
& =\frac{1}{2} \int_{0}^{2 T} S(\sigma) v d \sigma
\end{aligned}
$$

$v \in L^{2}(\Omega), c \in L^{2}(\Omega)$ であり, $\left\{\phi_{n}\right\}$ は $L^{2}(\Omega)$ で完備 正規直交基底をなすかから ${ }^{2)}$,

$$
\begin{array}{ll}
v=\sum_{n=1}^{\infty} v_{n} \phi_{n} \text { ここに } & v_{n}=\left\langle v, \phi_{n}\right\rangle_{L^{2}(\Omega)} \\
c=\sum_{n=1}^{\infty} c_{n} \phi_{n} & c_{n}=\left\langle c, \phi_{n}\right\rangle
\end{array}
$$

とフーリエ展開すると，(35）式により（36）式は，

$$
v_{n}+\frac{1}{2} \int_{0}^{2 T} e^{\lambda_{n} \sigma} d \sigma v_{n}=c_{n}, \quad n=1,2, \cdots
$$

となる。

$$
\therefore \quad v_{n}=\frac{c_{n}}{1+\frac{1-e^{-2(n \pi)^{2} T}}{2(n \pi)^{2}}}
$$

よって最適入力 $f^{\circ}$ は次式で与えられる。

$$
\begin{aligned}
f^{\circ}(t) & =\left(K_{1}^{*} v\right)(t)=S(T-t) v \\
& =\sum_{n=1}^{\infty} e^{-(n \pi) 2(T-t)} v_{n} \phi_{n} \\
& =\sum_{n=1}^{\infty} \frac{c_{n}}{1+\frac{1-e^{-2(n \pi) 2 T}}{2(n \pi)^{2}}} e^{-(n \pi)^{2}(T-t)} \phi_{n}
\end{aligned}
$$

\section{$5 \cdot 2$ 空間分布制御入力による最適追従問題}

(32) 式のシズテムに対して,

$$
\begin{aligned}
J(f) & =\frac{1}{2} \int_{0}^{T} \int_{0}^{1}\left|u(t, x)-u^{d}(t, x)\right|^{2} d x d t \\
& +\frac{1}{2} \int_{0}^{T} \int_{0}^{1}|f(t, x)|^{2} d x d t
\end{aligned}
$$

を最小にする入力 $f$ を決める。

$$
\left(K_{2} f\right)(t)=\int_{0}^{t} S(t-\sigma) f(\sigma) d \sigma, 0<t<T
$$

により, $L^{2}\left(0, T ; L^{2}(\Omega)\right)$ から $L^{2}\left(0, T ; L^{2}(\Omega)\right)$ への 有界作用素 $K_{2}$ 定義する. $K_{2}{ }^{*}$ は $L^{2}\left(0, T ; L^{2}(\Omega)\right)$ から自分自身への作用素であり，次式により定義され る。

$$
\begin{aligned}
&\left\langle K_{2} f, g\right\rangle_{L^{2}\left(0, T^{\prime} ; T^{2}(\Omega)\right)}=\left\langle f, K_{2}{ }^{*} f\right\rangle_{L^{2}\left(0, T^{\prime} ; L^{2}(\Omega)\right)}, \forall f \\
&\left\langle K_{2} f, g\right\rangle=\int_{0}^{T}\left\langle\left(K_{2} f\right)(t), g(t)\right\rangle_{L^{2}(\Omega)} d t \\
&=\int_{0}^{T} \int_{0}^{t}\langle S(t-\sigma) f(\sigma), g(t)\rangle d \sigma d t
\end{aligned}
$$

積分順序を交換すれば,

$$
=\int_{0}^{T}\left\langle f(\sigma), \int_{0}^{T} S(t-\sigma) g(t) d t\right\rangle d \sigma
$$$$
\therefore \quad\left(K_{2} * g\right)(\sigma)=\int_{\sigma}^{T} S(t-\sigma) g(t) d t
$$

$c(t)=u^{d}(t)-S(t) u(0) \in L^{2}(\Omega)$ と拝けば，最適解 $f^{\circ}$ は (23) 式から,

$$
\left[I+K_{2} * K_{2}\right] f^{\circ}=K_{2} * c
$$

である。まず $K_{2} * K_{2} f$ を計算する。

$$
\begin{aligned}
\left(K_{2} * K_{2} f\right)(t) & \equiv \int_{t}^{T} S(\sigma-t)\left(K_{2} f\right)(\sigma) d \sigma \\
& =\int_{t}^{T} S(\sigma-t)\left[\int_{0}^{\sigma} S(\sigma-\tau) f(\tau) d \tau\right] d \sigma \\
& =\int_{t}^{T} d \sigma \int_{0}^{\sigma} S(2 \sigma-t-\tau) f(\tau) d \tau
\end{aligned}
$$$$
f_{n}(\tau)=\left\langle f(\tau), \phi_{n}\right\rangle_{L^{2}(\Omega)}=\int_{\Omega} f(\tau, x) \phi_{n}(x) d x
$$

と护けば,

$$
=\sum_{n=1}^{\infty} \int_{t}^{T} d \sigma \int_{0}^{\sigma} e^{\lambda_{n}(2 \sigma-t-\tau)} f_{n}(\tau) d \tau \times \phi_{n}
$$

$c(t)$ のフーリエ係数を $c_{n}(t)$ として， $\left(23^{\prime}\right)$ 式の両 辺のフーリエ保数を比較すれば,

$$
\begin{aligned}
f_{n}(t) & +\int_{t}^{T} d \sigma \int_{0}^{\sigma} e^{\lambda_{n}(2 \sigma-t-\tau)} f_{n}(\tau) d \tau \\
& =\int_{t}^{T} e^{\lambda_{n}(\sigma-t)} c_{n}(\sigma) d \sigma
\end{aligned}
$$

$f_{n}(t)$ は $t$ について微分可能であることがわかり， （39）式を微分して整理すると次式を得る.

$$
\dot{f}_{n}(t)+\lambda_{n} f_{n}(t)-\int_{0}^{t} e^{\lambda_{n}(t-\tau)} f_{n}(\tau) d \tau=-c_{n}(t)
$$

この微分積分方程式を $f_{n}(T)=0$ のもとで解けば最適 入力 $f^{\circ}$ が決定される.

とくに， $c_{n}(t)$ が注とんどいたる所で微分可能なら ば（40）式は微分方程式にすることができ，陽の形に $f$ を求めることができる. $c_{n}(t)$ が微分可能な点では $\ddot{f}_{n}(t)$ が存在するから，

$$
\begin{aligned}
& \ddot{f}_{n}+\lambda_{n} \dot{f}_{n}-f_{n}-\lambda_{n} \int_{0}^{t} e^{\lambda_{n}(t-\sigma)} f_{n}(\sigma) d \sigma=-\dot{c}_{n} \\
& \therefore \quad \ddot{f}_{n}(t)-\left(1+\lambda_{n}{ }^{2}\right) f_{n}(t)=\lambda_{n} c_{n}(t)-\dot{c}_{n}(t)
\end{aligned}
$$
$\alpha_{n}=\sqrt{1+\lambda_{n}^{2}}$ と招いて (41) 式を解くと, $D_{n}, E_{n}$ を 積分定数として次式を得る。

$$
\begin{aligned}
& f_{n}(t)=D_{n} \sinh \alpha_{n} t+E_{n} \cosh \alpha_{n} t \\
&-\int_{0}^{t} c_{n}(s)\left[\cosh \alpha_{n}(t-s)\right. \\
&\left.-\frac{\lambda_{n}}{\alpha_{n}} \sinh \alpha_{n}(t-s)\right] d s \\
& \text { (40) 式から } \dot{f}_{n}(0)+\lambda_{n} f_{n}(0)=-c_{n}(0) \\
& \text { (39) 式から } f_{n}(T)=0
\end{aligned}
$$

(42) 式を満足するように, $D_{n}, E_{n}$ を決定すれば最終 
的に次式を得る.

$$
\begin{aligned}
f_{n}(t) & =\frac{\cosh \alpha_{n} t-\frac{\lambda_{n}}{\alpha_{n}} \sinh \alpha_{n} t}{\cosh \alpha_{n} T-\frac{\lambda_{n}}{\alpha_{n}} \sinh \alpha_{n} T} \int_{0}^{T} c_{n}(s) \\
& \times\left[\cosh \alpha_{n}(T-s)-\frac{\lambda_{n}}{\alpha_{n}} \sinh \alpha_{n}(T-s)\right] d s \\
& -\int_{0}^{t} c_{n}(s)\left[\cosh \alpha_{n}(t-s)\right. \\
& \left.-\frac{\lambda_{n}}{\alpha_{n}} \sinh \alpha_{n}(t-s)\right] d s
\end{aligned}
$$

よって最適入力 $f^{\circ}$ はつぎの式で与えられる.

$$
f^{\circ}(t, x)=\sum_{n=1}^{\infty} f_{n}(t) \phi_{n}(x)
$$

\section{6. 結言}

バナッ八空間にお乱る最適追従問題および最終值制 御問題を考察し，最適解の必要十分条件を導いた。ヒ ルベルト空間以外での最適制御問題の最適解は非線形 方程式で与えられることが示された。ヒルベルト空間 の場合についての例をあげ，それらの最適解を計算し た。な㧤 $J_{p, q}$ の最適制御（31）式を計算するにはた と党ば Altman ${ }^{15}$ の方法が適用できる. $L^{1}, L^{\infty}$ での 最適制御を与える式 (19)，(28) 式の計算法について は目下研究中である。

本稿の理論の展開からわかるよう飞，分布定数系を 半群の理論によってバナッハ空間に执いてダイナミカ ル表示し，これに基づいて最適制御問題を考察するこ とは理論的見通しをよくする，とくに有限次元で展開 された理論が自然の形で分布系に拡張されていること がわかる。な括本手法は結局入力-状態関係式が有界 作用素で表示できることを本質的に使っているだけで ある。したがって希望出力に追従させる問題について も，Kを入力-出力の作用素とするのみでそのまま適 用される。この点に関しては文献 $(16,17)$ でさらに 発展させた。

なお最適解を時間関数の形で求めたが，ヒルベルト 空間の場合には有限次元の場合によく知られているよ らにフィードバックの形に書くこともできる(11),18).

最後に, 本研究にさいして終始熱心に討論いただい た本学自動制御研究施設の諸氏, とくに助手松尾強, 大学院学生野村拓己, 赤塚義邦, 南出成康らの諸氏に 感謝する。

\section{参考 文 献}

1）舟橋・中村：半群の理論と放物型ダイナミカル・シス テムの解の存在と一意性, 名大自動制御研究施設報告, Vol.15, 8/14 (1968-4)

2） 舟橋・中村 : 分布定数系の入力・状態関係式のダイナ
ミカル表示, 同上 15/21

3) A. G. Butkovskii : Maximum principle for optimum systems with distributed parameters, Automation and Remote Control, 22-10, 1156/1169 (1962)

4) K. Ichikawa:Application of Pontryagin's maximum principle to the optimization of distributed parameter system, Res. Rept. of Aut. Cont. Lab., Faculty of Eng., Nagoya Univ., Vol. 15, 68/76 (1968-4)

5) P.K.C.Wang : Control of Distributed Parameter Systems, Advances in Control Systems, Vol. 1, Academic Press, 75/172 (1964)

6) H. O. Fattorini : Time-optimal control of solutions of operational differential equations, J.SIAM Control, 2-1, 54/59 (1964)

7) A.V.Balakrishnan: Optimal control problems in Banach spaces, J.SIAM Control, 3-1, 152/180 (1965)

8) A.Friedman : Optimal control in Banach spaces, J. Math. Anal. and Appl., 19-1, 35/55 (1967)

9) E.I. Axelband: A solution to the optimal pursuit problem for distributed parameter systems, J. Computer and System Sciences, 1-3, 261/286(1967)

10) D. M. Wiberg: Feedback control of linear distributed systems, J. of Basic Engineerings Ser. D., 89-2，379/384 (1967)

11）中村・舟橋：分布定数系のレギュレータ問題，第 6 回 計測制御学術講演会, 220 (昭 42.10 月)

12) N. Dunford and J. T. Schwartz : Linear Operators, part. 1 General Theory, Interscience Publ., 472 (1958)

13) F. A. Valentine: Convex Sets, $130 / 134$, McGraw-Hill Company (1964)

14) C. Berge : Espaces Topologiques, 198/204, (1966Dund)

15) M.Altman : On the Approximate Solutions of Operator Equations in $L^{p}$ Spaces, Bulletin de Academie Polonaise des Sciences, 5-12, 1099/1103 (1957)

16）中村・舟橋：二乗積分証価による最適制御問題，第 10 回自制連大, 110 (昭 42.11 月)

17）舟橋・中村：ヒルベルト空間に和ける最適追従問題 (「計測と制御」論文集投稿中）

18）舟橋・中村：ヒルベルト空間に捺ける最適フィードバ ック制御（投稿中）

$\square$ 付 録 $\square$

\section{I. $V(\epsilon)$ が $\epsilon>0$ について連続であること}

$$
\begin{aligned}
\mid V\left(\epsilon_{1}\right) & -V\left(\epsilon_{2}\right)|=| \frac{J\left(f+\epsilon_{1} g\right)}{\epsilon_{1}}-\frac{J\left(f+\epsilon_{2} g\right)}{\epsilon_{2}} \\
& -\left[\frac{J(f)}{\epsilon_{1}}-\frac{J(f)}{\epsilon_{2}}\right]|=| \frac{\left\|K f-c+\epsilon_{1} K g\right\|}{\epsilon_{1}} \\
& -\frac{\left\|K f-c+\epsilon_{2} K g\right\|}{\epsilon_{2}}+\frac{\left\|f+\epsilon_{1} g\right\|}{\epsilon_{1}}-\frac{\left\|f+\epsilon_{2} g\right\|}{\epsilon_{2}} \\
& -\left[\frac{J(f)}{\epsilon_{1}}-\frac{J(f)}{\epsilon_{2}}\right]|\leqq| \frac{1}{\epsilon_{1}}-\frac{1}{\epsilon_{2}} \mid\|K f-c\|
\end{aligned}
$$




$$
\begin{aligned}
& +\left|\frac{1}{\epsilon_{1}}-\frac{1}{\epsilon_{2}}\right|\|f\|+\left|\frac{1}{\epsilon_{1}}-\frac{1}{\epsilon_{2}}\right| J(f) \\
& \leqq 2\left|\frac{1}{\epsilon_{1}}-\frac{1}{\epsilon_{2}}\right| J(f)
\end{aligned}
$$

II. $\boldsymbol{L}_{1}(\boldsymbol{0}, \boldsymbol{T})$ のノルムの片側ガトウ微分*

$$
\begin{aligned}
& Z(f)=\{t \in(0, T) \mid f(t)=0\}, \\
& R(f)=\{t \in(0, T) \mid f(t) \neq 0\}
\end{aligned}
$$

$\operatorname{sgn} f(t)=\left\{\begin{array}{cc}f(t) / f(t), & f(t) \neq 0 \\ 0, & f(t)=0\end{array}\right.$ と定義する.

$$
\begin{aligned}
\lim _{\varepsilon \downarrow 0} & \frac{\|f+\epsilon g||-\| f \|}{\epsilon} \\
& =\lim _{\varepsilon \downarrow 0} \int_{0}^{T} \frac{|f(t)+\epsilon g(t)|-f(t) \mid}{\epsilon} d t \\
& =\lim _{\varepsilon \downarrow 0}\left[\int_{Z(f)} \frac{|\varepsilon g(t)|}{\varepsilon} d t\right. \\
& \left.+\int_{R(f)} \frac{1}{\varepsilon} \frac{|f(t)+\epsilon g(t)|^{2}-|f(t)|^{2}}{|f(t)+\epsilon g(t)|+|f(t)|} d t\right] \\
& =\int_{Z(f)}|g(t)| d t \\
& +\lim _{\varepsilon \downarrow 0} \int_{R(f)} \frac{2 f(t) g(t)+\epsilon|g(t)|^{2}}{|f(t)+\epsilon g(t)|+|f(t)|} d t \\
& =\int_{Z(f)}|g(t)| d t+\int_{R(f)} \frac{f(t)}{|f(t)|} g(t) d t \\
& =\int_{Z(f)}|g(t)| d t+\int_{0}^{T} \operatorname{sgn} f(t) \cdot g(t) d t \\
& =\int_{Z(f)}|g(t)| d t+\langle\operatorname{sgn} f, g\rangle
\end{aligned}
$$

$Z(f) \cap R(g)$ のルベーク測度がゼロなるときは，

$$
=\langle\operatorname{sgn} f, g\rangle
$$

したがって， $Z(f)$ の測度がゼロなる $f$ の点では $L^{1}$ のノルムはガトウ微分可能である.

$f$ の值域が例えば $L^{1}(\Omega)$ なるときにはつぎのよう になる。

$$
\begin{aligned}
& Z(\dot{f})=\{(t, x) \in(0, T) \times \Omega \mid f(t, x)=0\} \\
& R(f)=\{(t, x) \in(0, T) \times \Omega \mid f(t, x) \neq 0\}
\end{aligned}
$$

$$
\||f|\|=\int_{0}^{T}\|f(t)\|_{L^{1}(\Omega)} d t=\int_{0}^{T} \int_{\Omega}|f(t, x)| d x d t
$$

$$
\begin{aligned}
\therefore & \lim _{\varepsilon \downarrow 0} \frac{\||f+\epsilon g\|\|-\||\|f\||}{\epsilon} \\
= & \iint_{Z(f)}|g(t, x)| d x d t \\
& +\int_{0}^{T} \int_{\Omega} \operatorname{sgn} f(t, x) g(t, x) d x d t
\end{aligned}
$$

\section{III. $\left[\boldsymbol{I}+\boldsymbol{K}^{*} \boldsymbol{K}\right]$ の作用素に関する諸点の証明}

[1] $I+K * K$ の逆作用素が存在すること.

$(I+K * K) f=0$ をみたす $f$ が 0 元に限ることを示 せばよい。

$$
0=\left\langle f,\left(I+K^{*} K\right) f\right\rangle=\|f\|^{2}+\|K f\|^{2}
$$

$\|K f\|^{2} \geqslant 0$ であるから $\|f\|=0$ すなわち $f=0$ である。

[2] $\left[I+K^{*} K^{-1}\right]^{-1}$ が有界作用素であること。

* B.K. Kripke and T. J. Rivin : Approximation in the metric of $L^{1}(X, \mu)$, Trans. Amer. Math. Soc., 119, $101 / 122$ (1965)
$f=[I+K * K]^{-1} g$ と和くと, $g=(I+K * K) f$ であ るから，

$\|g\|^{2}=\left\|f+K^{*} K f\right\|^{2}=\|f\|^{2}+2\|K f\|^{2}$

$$
+\left\|K^{*} K f\right\|^{2} \geqq\|f\|^{2}=\left\|\left(I+K^{*} K\right)^{-1} g\right\|^{2}
$$

すなわち，

$$
\left\|\left[I+K^{*} K\right]^{-1} g\right\| \leqq\|g\|, \quad \forall g
$$

よって $\left[I+K^{*} K\right]^{-1}$ は有界作用素であり，作用素， ルムは 1 でおさ方られる。

[3] $\left[I+K^{*} K\right]^{-1} K^{*}=K^{*}\left[I+K K^{*}\right]^{-1}$ であるこ c.

$$
\begin{aligned}
& f=\left[I+K^{*} K\right]^{-1} K^{*} c \\
& g=K^{*}\left[I+K K^{*}\right]^{-1} c
\end{aligned}
$$

とおく. $f, g$ に $\left(I+K^{*} K\right)$ を作用させると，

$$
\left[I+K^{*} K\right] f=K^{*} c
$$$$
\left[I+K^{*} K\right] g=\left[I+K^{*} K\right] K^{*}\left[I+K K^{*}\right]^{-1} c
$$

$$
=K^{*}\left[I+K K^{*}\right]\left[I+K K^{*}\right]^{-1} c
$$

となる。

$$
\begin{aligned}
\therefore \quad\left[I+K^{*} K\right] f & =\left[I+K^{*} K\right] g \\
& \text { i.e. } \quad f=g
\end{aligned}
$$

IV. $\boldsymbol{L}^{p}(\boldsymbol{0}, \boldsymbol{T}) 1<\boldsymbol{p}<\infty$ でのノルムの微分

$$
\begin{aligned}
\lim _{\varepsilon \downarrow 0} & \frac{\left\|f+\epsilon g||^{p}-\right\| f \|^{p}}{\epsilon} \\
& =\lim _{\epsilon \downarrow 0} \frac{1}{\epsilon} \int_{0}^{T}\left[|f(t)+\epsilon g(t)|^{p}-|f(t)|^{p}\right] d t \\
& =\lim _{\epsilon \downarrow 0} \int_{0}^{T}\left\{|f(t)+\epsilon g(t)|^{p-1}+\cdots\right. \\
& \left.+|f(t)|^{p-1}\right\} \frac{|f(t)+\epsilon g(t)|-|f(t)|}{\epsilon} d t \\
& =\lim _{\epsilon \downarrow 0} \int_{Z(f)}|g(t)|^{p} d t \varepsilon^{p-1} \\
& +\lim _{\epsilon \downarrow 0} \int_{R(f)}\{l \mid\} \frac{|f(t)+\epsilon g(t)|^{2}-|f(t)|^{2}}{\varepsilon[|f(t)+\epsilon g(t)|+|f(t)|} d t \\
& =\int_{R(f)} p|f(t)|^{p-1} \operatorname{sgn} f(t) g(t) d t \\
& =\int_{0}^{T} p \mid f(t)^{p-1} \operatorname{sgn} f(t) g(t) d t \\
& =\left\langle p|f|^{p-1} \operatorname{sgn} f ; g \backslash\right.
\end{aligned}
$$

とくに $p=2$ の場合には,

$$
=2\langle f, g\rangle
$$

である．gについて線形であるから， $L^{p}(0, T), 1<$ $p<+\infty$ のノルムはガトウ微分可能である. $L^{p}(0, T$; $\left.L^{p}(\Omega)\right)$ について子同様の式を得る.

V.

$(0, T)$ 上で定義された有界関数に $\|f\|_{\infty}=\sup _{0 \leq t \leq T}|f(t)|$ なるノルムを導入した空間での片側微分*

$$
\begin{aligned}
& \lim _{\epsilon \downarrow 0} \frac{\|f+\epsilon g\|_{\infty}-\|f\|_{\infty}}{\varepsilon} \\
& \quad=\inf _{\epsilon>0}\left[\sup _{|\|f\|-f(t)|<\epsilon} f(t) g(t)\right]
\end{aligned}
$$

とくに $g$ が連続関数であるときには，

$$
=\max _{t:|f(t)|=\|f\|_{\infty}} f(t) g(t)
$$

* V.L.Smulian : Sur la structure de la sphère unitaire dans l'espace de Banach, Matemathueckий Сборник T. 9 (51), N 3, 545/561 (1941) 